\title{
PID fuzzy control applied to an electrosurgical unit for power regulation
}

\author{
Ali Mohammed Ridha ${ }^{1}$, Ali Jafer Mahdi ${ }^{2,5}$, Jameel Kadhim Abed ${ }^{3}$, Shah Fahad ${ }^{4}$ \\ 1. College of Medicine, University of Al-Ameed, Karbala, Iraq \\ 2. Department of Electrical and Electronics Engineering, University of Kerbala, Karbala, Iraq \\ 3. Department of Medical Instrumentation Techniques Engineering, Middle Technical University, Baghdad, Iraq \\ 4. Department of Electrical Engineering, Zhejiang University, P.R of China \\ 5. E-mail any correspondence to: ali.j.mahdi@uokerbalaedu.iq
}

\begin{abstract}
The electrosurgical unit (ESU) is the most common device in modern surgery for cutting and coagulation of tissues. It produces high-frequency alternating current to prevent the stimulation of muscles and nerves. The commercial ESUs are generally expensive and their output power is uncontrolled. The main objective of the proposed study is to propose an economic ESU with an additional feature of output power regulation using a fuzzy logic controller (FLC) based proportional integral derivative (PID) tuned controller. Unlike the previous studies, the proposed controller is designed in a fully closed-loop control fashion to regulate the output power of the ESU to a fixed value under the consideration of highly dynamic tissue impedance. The performance of the proposed method is tested in the MATLAB/SIMULINK environment. In order to validate the superiority of the proposed method, a comparative analysis with a simple (PID) controller based ESU is presented.
\end{abstract}

Keywords: Electrosurgical unit; tissue burning; tissue impedance; boost converter; high-frequency inverter

\section{Introduction}

Power electronics plays an important role in medical devices. These kinds of electronic equipment may include either wired or wireless power transfer depending on application. An example is the MRI machine, which is one of the most advanced diagnostic instruments [1-3]. Several applications of power electronics include electro-cautery, electroportion, muscle stimulation, and electro-surgical unit (ESU), which are usually in direct contact with tissues. ESU is a common device used to execute arc coagulation and cutting tissues in modern surgery [4]. An ESU uses alternating current $(A C)$ of a high frequency (i.e. $100-500 \mathrm{kHz}$ ) to excite a clinical reaction in tissue using a plasma arc. A standard ESU is dependent on a resonant inverter, which works to regulate the wave shape and output frequency [5].

Adopting high frequencies to prevent unwanted nerve and muscle stimulation is harmful for the patient [6]. An ESU surgery takes place when the current produced by the generator, is penetrating into the patient's tissue using an active electrode and exits through the neutral electrode [7].

As illustrated in Figs. 1 and 2, the ESU operates in either monopolar or bipolar mode of operation [8]. In monopolar ESU, the high current density concentrates at the active electrode tip before cutting the patient's body, which then behaves as a part of the electric circuit. The current drains from the active electrode at the treatment site through the patient's body and then return to the neutral electrode as explained in Fig. 1.

The electric current leaves the patient's body through a relatively large passive electrode that is dispersed over a large area and is considered a low current density area. In bipolar ESU, this instrument is similar to forceps. It consists of a pair of electrodes on both sides. These electrodes have similar size and construction with pointy tips so that the current can pass between them in a closed circuit. The only tissue exposed to the electric current is the tissue grasped by the forceps, as shown in Fig. 2. Fig. 2 shows that the bipolar ESU is less hazardous than monopolar $\operatorname{ESU}[8,9]$. 


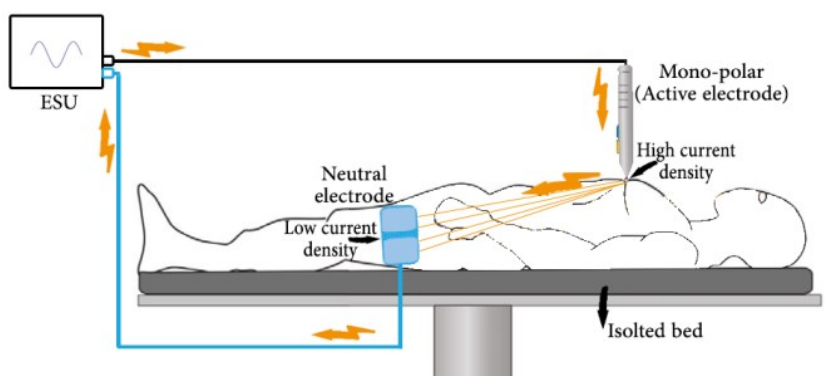

Fig.1: Mono-polar ESU Modes.

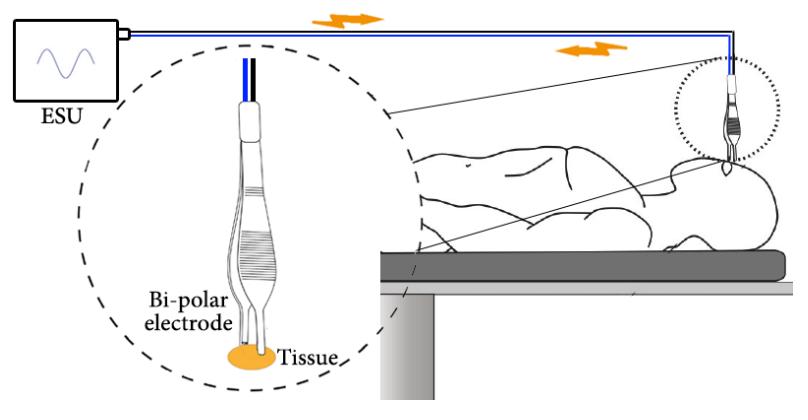

Fig.2: Bi-polar ESU Modes.

The ESU is required to vary the output electrical parameters according to the desired effect. The operations performed by the ESU depend on the power of the output waveform, such as cutting, coagulation or blended. Here, the operational modes are described with crest factor (CF) [10]. ESU techniques compared to other surgical instruments such as laser surgery, should have the ability to rapidly cut tissue and decrease blood loss and vascular clotting [11]. The power that must be used in surgical operations, despite the difference in tissue resistance, ranges between 5 and $100 \mathrm{~W}$.

According to the tissue impedance, which ranges between 130 and $340 \Omega[6,12]$, adjustment of power magnitude is performed manually depending on operator experience. The magnitude of power generally depends on the type of tissue, electrode, speed of electrode and proposed amount of hemostasis [13]. The simple mechanism for cutting or coagulation tissue in ESU involves Joule heating of tissue by passing electric current. This will lead to the vaporization of water contents and ionize the tissues adjacent to the active electrode. The distribution of electric field (which is the source of heating) with thermal diffusion is a way to determine the heat penetration depth into the tissue. Thus, to limit the damage in tissue, thermal diffusion should be controlled to prevent tissue burning [14]. Smoke from surgical instruments is generated when the thermal function of the ESU creates arcs leading to vaporization of tissue by. The entities present in this smoke include bacteria, DNA fragments and carcinogens [15].

Tissue burns is the most common problem faced in surgeries with high frequency ESU. As a result, the decrease in thermal cuts caused by an ESU, represents a significant progress of the medical field [16]. Modern developments to regulate the output power of ESUs depends on observing the tissue impedance. For keeping a constant power for coagulation and cutting under varying circumstances at the electrode, power should be consistently regulated even for changes in impedance due to the tissue interface [14]. Recently, above $90 \%$ of all surgical procedures utilize ESU. Regardless of the fast progress and implementation of new methods, the use of ESUs brings many hazards for the patient, which might lead to fatal penalties [17].

The impedance of tissue varies with different parts of the body. Interference of varying impedance of tissue with the high-power currents of radio frequency delivered at the output terminal of ESU, tends to keep varying the output power. The change in the ESU output power should be planned in such a manner that it copes with the uncertainty of tissue impedance and delivers power at the specific level. In addition, the power variation due to varying voltage and current should also be considered [18].

In [6], the authors proposed a fly-back converter with a single-phase inverter based on IGBT for high-power density implementation, which enhanced the performance of ESU. This converter offers voltage control for the surgical operation. In [10], the authors proposed a new topology of a boost converter (step-up) containing Gallium Nitride (GaN) devices to deliver a high-frequency ESU. The converter allows producing an adaptable wave shape with necessary requirement through the application of controlling the voltage according to the requirements. The authors in [12] proposed an electrical comparable circuit for tissue simulation in ESU models and tested it using the frequency curves versus impedance measured in muscle tissue and porcine liver. The circuit offers precise simulation of tissue impedance in the frequency range from $1 \mathrm{kHz}$ to $1 \mathrm{MHz}$. In [13], the philosophy of slicing or cutting is assessed, and objectives are achieved to control the effects of an active electrode speed with generator-controlled technique. In all these studies, the focus is based on either the voltage regulation or improved frequency operation, however, the power regulation has not been discussed.

In [16], the authors proposed an accelerometer and gyroscope based portable emissions measurement system technology to correct the output power of the ESU according to the speed of the active electrode. In [26], the authors suggested that the ESU can measure the active power in realtime that is delivered to the patient during surgery. The results indicated an error rate of less than $5 \%$, and it succeeded to regulate the power of the ESU. The authors in [27] suggested an ESU model that can control temperature by means of a heating and cooling system with output power regulation in order to avoid side damage to the tissues during the surgery. In [28], the authors proposed a new circuit to regulate the output power of the ESU to a constant value. 


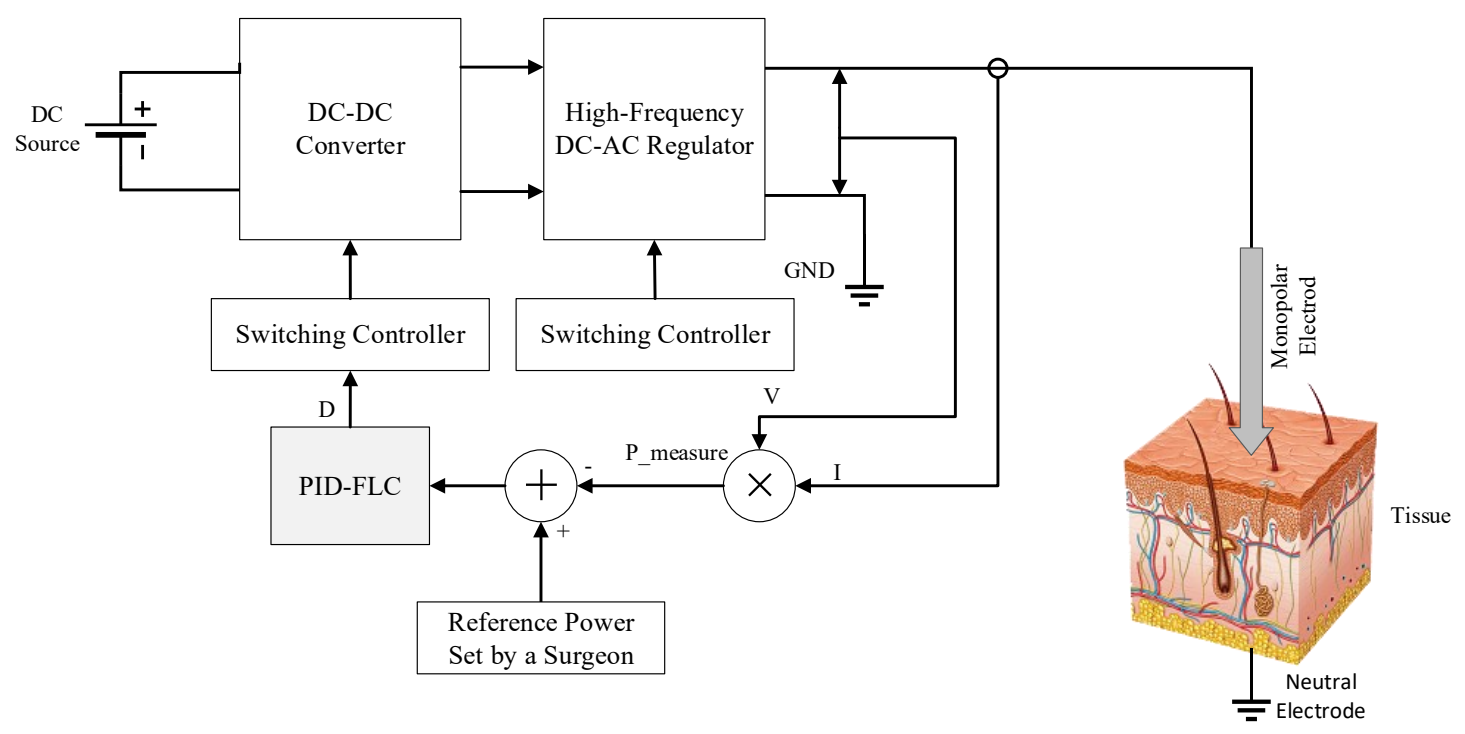

Fig.3: Block diagram of the proposed ESU.

This circuit successfully regulates the output power using PID controller based sliding mode control, which successfully reduces the risk of tissue damage as compared to previous methods. In all these studies, the power output regulation is discussed, however, the cost of the controller parameters along with the advanced artificial intelligence-based tuning, is neglected.

In this study, the main contribution is to propose a modified control strategy for ESUs, which involves a PID controller with an artificial intelligence based Fuzzy Logic Controller (PID-FLC) for power regulation of a low cost ESU to minimize the thermal damage that occurs in the patient's tissue during surgical operation. The major contributions of the proposed study are:

- To present an artificial intelligence-based tuning for PID controller introduced for the first time in ESU.

- Unlike the previous studies, the proposed work also contributes towards the cost analysis of ESU.

- To present a comparative analysis of the proposed method with other conventional methods in order to validate its superiority.

\section{Materials and methods}

Block diagram of the proposed ESU

In this project, a control technique is suggested based on a schematic diagram illustrated in Fig. 3. This technique consists of a DC-DC buck converter, high-frequency inverter, FLC and PID controller, which will be explained in the later sections. The main focus of this paper is to regulate the power produced by the ESU inside the patient's body during surgical operation by controlling the voltage independent on any change in tissue impedance in skin layers.

\section{The load (tissues)}

In general, biological tissues consist of three layers: skin, fat, and muscle. These layers have different electrical impedance depending on the patient's sex, body and type of tissue [19]. Although the surgeon sets the appropriate power for operation, e.g. $50 \mathrm{~W}$, due to the gradual decrease/increase in the resistance between the layers of the skin, the output power of the ESU varies accordingly. The equation of relation of power to the tissue resistance is given in Eq. (1), where $P$ is the ESU output power, $V$ is the ESU voltage, and $R$ is the tissue impedance. The impedance variation leads to a high temperature rise in the tissue, which eventually leads to carbonization of the tissues.

$$
P=\frac{V^{2}}{R}
$$

In order to prevent the effect of impedance variation, as mentioned earlier, the output power must be regulated to a fixed value according to the desired value by the surgeon. In this work, an equivalent electrical circuit is used with the same electrical properties as biological tissue [12]. In this study, the magnitude of the tissue's impedance $|Z|$ varies by $30 \Omega$ for each layer, where the range of $|Z|$ is $160-220 \Omega$, as illustrated in Fig. 4 [12].

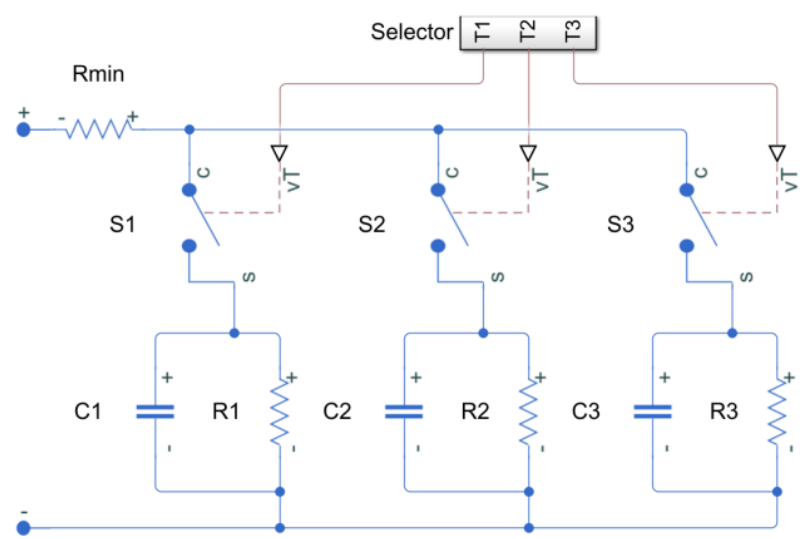

Fig.4: Equivalent electric circuit of a biological tissue. 


\section{Power regulation}

In this paper, the output power regulation technique is implemented to minimize thermal damage. To prevent the tissue from burning, voltage is controlled via pulse width modulation (PWM) using switch-mode converters (SMC). The SMC requires a signal with a constant high frequency while its ON and OFF times are varied to achieve desired closed-loop operation. The time of the PWM signal remains constant, however, the duty cycle (i.e. the ratio of ON time to the time period) is adjusted to achieve the desired voltage [20]. In PWM1, the duty cycle is variable and controlled by a microcontroller to obtain a suitable voltage level at the output of the buck converter, which is connected directly to the inverter. In PWM2, the duty cycle is fixed at $50 \%$ to obtain a symmetric square wave signal driving the inverter.

Due to the variation of tissue impedance, the buck converter's output voltage must be controlled via a PID controller to follow the reference voltage, which is generated from the power control loop. Even if there is variation in human tissue impedance, constant power output is to be maintained. The PID controller in the time domain is given as:

$$
u(t)=k_{\mathrm{p}} e(t)+k_{\mathrm{i}} \int_{t_{\mathrm{o}}}^{t} e(t) d t+k_{d} \frac{d e(t)}{d t}
$$

where, $\mathrm{u}(\mathrm{t})$ is signal output, $\mathrm{e}(\mathrm{t})$ is signal error, $k_{p}$ is proportional coefficient, $k_{i}$ is integral coefficient and $k_{d}$ is derivative coefficient. The mathematical equation of the PID controller is described in the frequency domain. The Transfer Function (TF) of the PID controller is presented as follows [21]:

$$
\mathrm{TF}=\frac{u(s)}{E(s)}=k_{p}+\frac{k_{i}}{s}+k_{d} s=\frac{k_{d} s^{2}+k_{p} s+k_{i}}{s}
$$

In this project the authors used two different methods for tuning $k_{p}, k_{i}$ and $k_{d}$ of the PID controller, which are discussed in the sections below.

\section{Ziegler Nichols strategy (ZNS)}

Ziegler Nichols strategy (ZNS) depends on the idea of tuning the PID controller using the simple properties of step response [22]. Fig. 5. shows PID based ZNS for the proposed ESU. The steps for tuning a PID controller are: 1) Reduce the integrator and differentiator gains to zero; 2 ) Increase $k_{p}$ from zero to some critical value $k_{c r}=k_{p}$ at which the oscillations are sustained; 3 ) Record the value of $k_{\mathrm{cr}}$ and the corresponding period for sustained oscillations i.e. $P_{c r}$. Finally, calculate $k_{p}, T_{i}$, and $T_{d}$ from the equations below.

$$
\begin{aligned}
& k_{\mathrm{p}}=0.6 k_{\mathrm{cr}} \\
& T_{i}=\frac{P_{\mathrm{cr}}}{2}
\end{aligned}
$$

$$
T_{\mathrm{d}}=\frac{P_{\mathrm{cr}}}{8}
$$

Then calculate $k_{i}$ and $k_{d}$ from the equations below.

$$
\begin{aligned}
& k_{\mathrm{i}}=\frac{k_{\mathrm{p}}}{T_{\mathrm{i}}} \\
& k_{\mathrm{d}}=k_{\mathrm{p}} * T_{\mathrm{d}}
\end{aligned}
$$

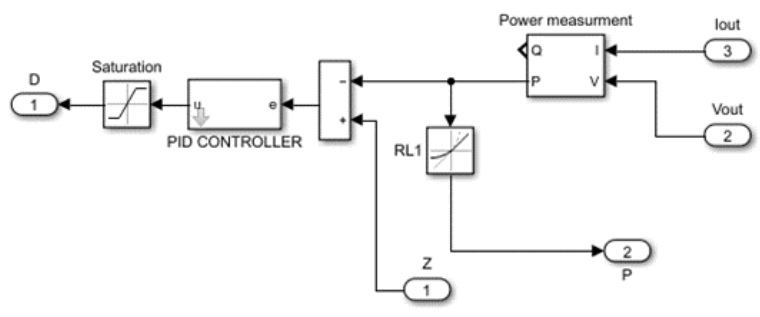

Fig.5: PID-ZNS controller for the proposed ESU.

\section{Fuzzy logic controller (FLC)}

The typical coefficients are not optimally selected so that the dynamic response of the ESU needs improvement using the proposed PID-FLC. Adding a fuzzy control strategy to the typical PID controller can enhance the flexibility and improve the rise time and overshoots under the variation of the tissue's impedance, as shown in Fig. 6 [23].

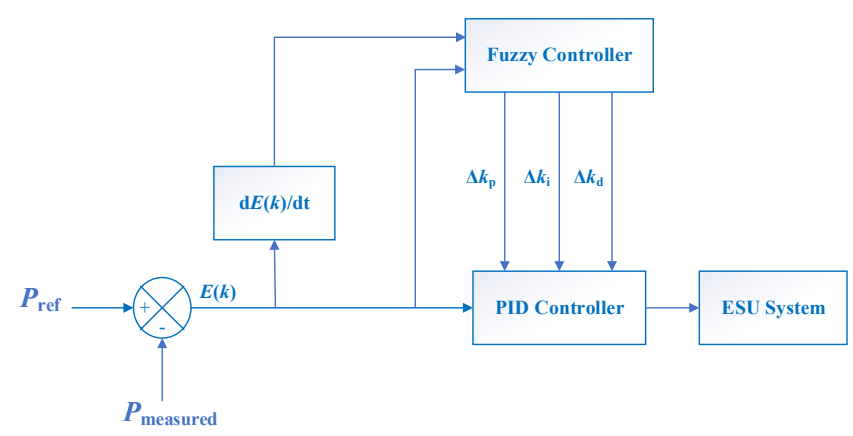

Fig.6: FLC-PID controller for the proposed ESU.

The steps for tuning the PID controller by using FLC are as follows; (1) Calculate the error signal in output power $E(k)$, which is the difference between the power reference $\left(P_{\text {ref }}\right)$ specified by the surgeon and the real power $\left(P_{\text {measured }}\right)$ delivered to the tissues; (2) Calculate the derivative of error $\Delta E(k)$, which is the change in the error signal of the output power.

Here, $E(k)$ and $\Delta E(k)$ are the inputs of the FLC, which are obtained from Eqs. (9) and (10). The $\Delta k_{p}, \Delta k_{i}$ and $\Delta k_{d}$ are the outputs of the FLC.

$$
\begin{gathered}
E(k)=P_{\text {ref }}-P_{\text {measured }}(k) \\
\Delta E(k)=E(k)-E(k-1)
\end{gathered}
$$


The FLC has been implemented from two inputs. Each input has seven member functions (MFs), so forty-nine rules (MFs $\wedge$ no. of inputs) must be built to adjust the PID parameters (FLC outputs). Here, $\Delta k_{p}, \Delta k_{i}$ and $\Delta k_{d}$ are the change in the parameters of the PID controller. Furthermore, $k_{\text {pref }}, k_{\text {iref }}$ and $k_{\text {dref }}$ are the PID parameters that are calculated by the ZNS. $k_{\text {pnew }}, k_{\text {inew }}$ and $k_{\text {dnew }}$ are the new PID parameters that are calculated by the FLC, and which are obtained from Eqs. (11, 12, and 13).

$$
\begin{aligned}
& k_{\text {pnew }}=k_{\text {pref }}+\Delta k_{\mathrm{p}} \\
& k_{\text {inew }}=k_{\text {iref }}+\Delta k_{\mathrm{i}} \\
& k_{\text {dnew }}=k_{\text {dref }}+\Delta k_{\text {d }}
\end{aligned}
$$

The gains of the PID controller should be calculated to maintain the appropriate value of the duty cycle $(D)$ of the buck converter. Thus, power is automatically adjusted according to the change in tissue impedance to track the reference power.

\section{$D C$-to-DC buck converter}

The DC chopper used in the proposed circuit is illustrated in Fig. 7.

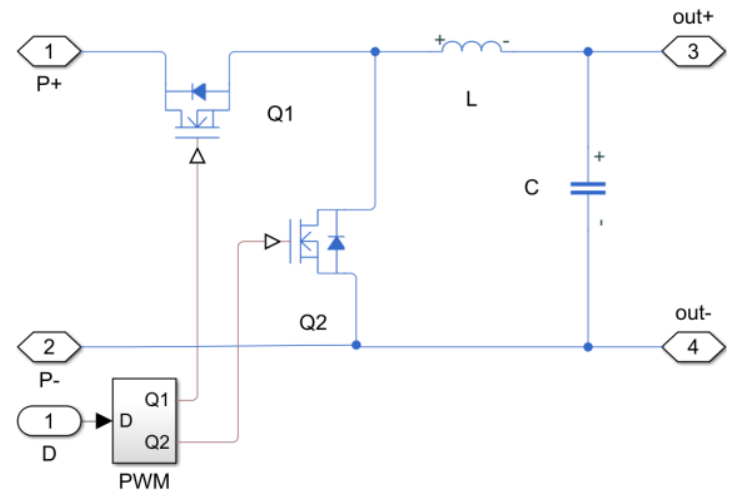

Fig.7: Buck converter for proposed ESU.

It is used to produce a regulated variable $D C$ voltage depending on the type of application. A detailed explanation of the DC chopper can be found in [24]. The mathematical equations for the buck converter are:

$$
\begin{aligned}
& V_{o}=D * V_{S} \\
& D=\frac{T_{o n}}{T} \\
& C_{\text {min }}=\frac{(1-D) T^{2}}{8 * L\left(\frac{\Delta V_{\circ}}{V_{\circ}}\right)} \\
& L_{\text {min }}=\frac{R(1-D) T}{2}
\end{aligned}
$$

$T_{\text {off }}$ is the time interval when the switch $S$ is OFF, $T_{\text {on }}$ is the time interval once the switch $\mathrm{S}$ is $\mathrm{ON}$, and $T$ is the total time.
The duty cycle $(D)$ of the buck converter is used to regulate voltage, which allows the DC output voltage to be regulated against changes in the load resistance and the DC input voltage. The minimum values of the capacitance and the inductance of the DC buck converter are determined from Eqs. (16 and 17), respectively. In simulations, these values are increased to verify the operation of the buck converter in continuous conduction mode as well as to improve the reliability of the device under the variation of load impedance.

\section{High-frequency inverter}

The high-frequency inverter, shown in Figure 8 , is used to convert the DC current to high frequency $A C$ current. The principle of operation of this circuit is to feed current into common ground. Two centers tapped transformers using two switches (transistors) work alternately. A symmetric square wave signal drives these transistors. When the transistor $Q_{1}$ is switched $\mathrm{ON}$ for a time $T_{o} / 2$, the voltage induced in the secondary coil is equal to $k v_{s}$. If the transistor $Q_{2}$ is switched ON for a time $T_{o} / 2$, the voltage across the secondary coil is $-k v_{s}$. Here, $k$ is the turns ratio and $V_{s}$ is the source voltage [25]. The inverter's output is attached to the active electrode via an HF transformer that interacts with the patient tissue for surgical operation.

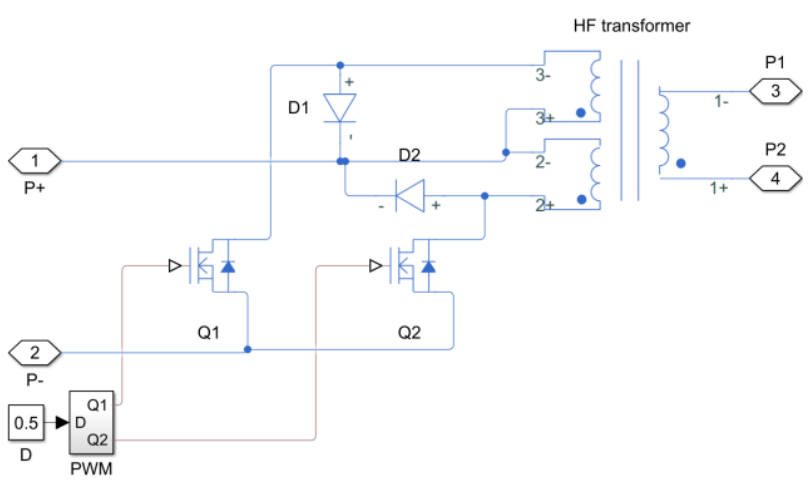

Fig.8: High-frequency inverter for the proposed ESU.

\section{Ethical approval}

The conducted research is not related to either human or animal use.

\section{Results and discussions}

The proposed strategy is implemented in the MATLAB/ SIMULINK environment and the simulation specifications are listed in Table 1 . To validate the proposed controller, simulation results are presented in Figs. 9-15. In the proposed system, the time response of voltage, current and power under various scenarios, i.e. fixed and variable tissue impedance, is presented.

It is clear from Fig. 7 that reduction in DC power can be achieved by adjusting $D$. In the proposed controller (PIDFLC), the parameters of the PID controller are optimally tuned based on the error and derivation error signals. The 
FLC contains two inputs, which are $E(k)$ (from Eq.(9)) and $\Delta E(k)$ (from Eq.(10)). For estimating the optimal constants of the PID controller, which are $k_{p}, k_{i}$ and $k_{d}$, the interior structure of the FLC includes forty-nine rules. The rules were created according to the inputs for each output. The range of outputs of the FLC are: $k_{p}=(0-0.005), k_{i}=(0-0.15)$ and $k_{d}=$ $\left(0-1 \mathrm{e}^{-7}\right)$. The initial values of $k_{p}, k_{i}$, and $k_{d}$ are estimated using the ZNS technique. In this paper, the equivalent circuit (as shown in Fig. 4) of the tissue is represented by three resistors connected in parallel with three capacitors, which represent the layers of the skin. The step-change is implemented in tissue impedance every $0.01 \mathrm{~s}$ using relays. For the first layer, the impedance value is $220 \Omega$ at the time $(0-0.01) s$ using the first relay. For the second layer, the impedance value is $190 \Omega$ at the time (0.01-0.02)s by enabling the second relay. For the third layer, the impedance value is $160 \Omega$ at the time (0.02-0.03)s when operating the third relay. The gradual decline in tissue impedance is due to the presence of liquid substances that increase conductivity and reduce impedance.

It is a common knowledge that to maintain power, either voltage or current has to be compensated. In this study, the same phenomenon is used to regulate the output power of the ESU. The control methods in open-loop (original/ conventional controller) and closed-loop (ZNS and FLC) are compared to show the superiority of the proposed PID-FLC.

In Figs. 9 and 10, the voltage graph for the original controller and both tuning methods of the PID controller (ZNS and FLC) are presented, respectively. It can be observed that, using the first relay when the impedance is $220 \Omega$, the output voltage of the original controller is not smooth compared to the closed-loop controllers. The transient response of the open-loop control is faster as compared to the closed-loop controller.

The reason for the fast response is that an open-loop control does not need to follow any reference command. In contrast, a closed-loop control does not settle unless the reference command is fulfilled, which increases its percentage of overshoot (OS\%). After the impedance is reduced with relays 2 and 3, the output voltage of the original controller slightly reduces in response to an increase in load. On the other hand, closed-loop controllers in Figure 10 reduces the voltage to a level where the output power of ESU is maintained to the reference command.

This theoretical analysis also implies to current and power analysis. The OS\% for original controller is the lowest among all three controllers because it is an open-loop system and it does not focus on a specific reference-based goal. The OS\% and the average standard deviation (STD) for the three controllers are illustrated in Table 2. The FLC-PID controller proposed in this study reduces the OS\% and STD than the PID-ZNS controller.
Table 1: Simulation specifications of the proposed ESU.

\begin{tabular}{|c|c|c|}
\hline \multicolumn{2}{|c|}{ Description Symbol } & Value, Unit \\
\hline \multicolumn{2}{|c|}{ Source Voltage } & $24 \mathrm{~V}$ \\
\hline \multicolumn{3}{|c|}{ Buck Converter } \\
\hline \multicolumn{2}{|c|}{$L$} & $33 \mu \mathrm{H}$ \\
\hline \multicolumn{2}{|c|}{$C$} & $220 \mu \mathrm{F}$ \\
\hline \multicolumn{2}{|c|}{ Switching frequency } & $500 \mathrm{kHz}$ \\
\hline \multicolumn{3}{|c|}{ Tissue Impedance } \\
\hline \multicolumn{2}{|c|}{$1^{\text {st }}$ layer, $Z_{1}$} & $90 \Omega$ \\
\hline \multicolumn{2}{|c|}{$2^{\text {nd }}$ layer, $Z_{2}$} & $60 \Omega$ \\
\hline \multicolumn{2}{|c|}{$3^{\text {rd }}$ layer, $Z_{3}$} & $30 \Omega$ \\
\hline \multicolumn{2}{|c|}{$Z_{\min }$} & $130 \Omega$ \\
\hline \multicolumn{3}{|c|}{ PID Parameters } \\
\hline$k_{p}$ & \multirow{3}{*}{$\begin{array}{c}\text { ZNS } \\
\text { method }\end{array}$} & 0.0102 \\
\hline$k_{i}$ & & 13.16 \\
\hline$k_{d}$ & & $1.98 \mathrm{e}-6$ \\
\hline$k_{\text {pref }}$ & \multirow{6}{*}{$\begin{array}{c}\text { FLC } \\
\text { method }\end{array}$} & 0.0102 \\
\hline$k_{\text {iref }}$ & & 13.16 \\
\hline$k_{\text {dref }}$ & & $1.98 \mathrm{e}-6$ \\
\hline$k_{\text {pnew }}$ & & 0.0114 \\
\hline$k_{\text {inew }}$ & & 13.197 \\
\hline$k_{\text {dnew }}$ & & $2.005 e-6$ \\
\hline \multicolumn{2}{|c|}{ Power Reference } & $50 \mathrm{~W}$ \\
\hline \multicolumn{3}{|c|}{ High-Frequency Transformer } \\
\hline \multicolumn{2}{|c|}{$L_{1}$} & $40 \mu \mathrm{H}$ \\
\hline \multicolumn{2}{|c|}{$L_{2}$} & $0.1 \mu \mathrm{H}$ \\
\hline \multicolumn{2}{|c|}{$L_{3}$} & $0.1 \mu \mathrm{H}$ \\
\hline
\end{tabular}

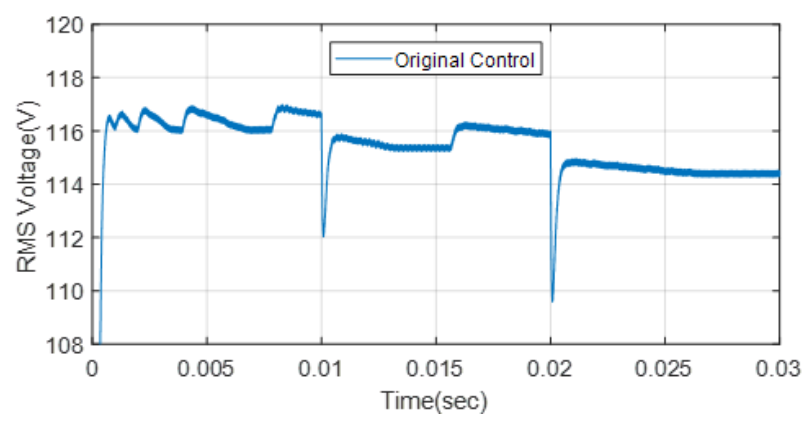

Fig.9: ESU output RMS voltage for original controller.

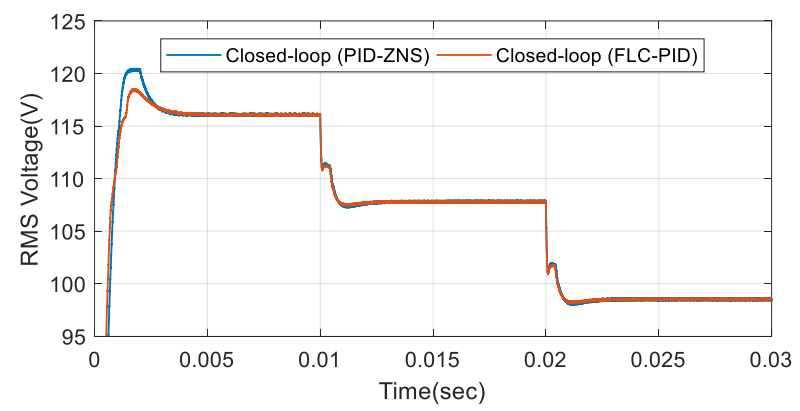

Fig.10: RMS output voltage for proposed ESU with PID-ZNS and FLC-PID controller.

In Fig. 11 and 12, comparison of the current signal for the original controller and for two closed-loop methods of PID controller tuning (ZNS and FLC) is presented, respectively. As previously discussed, the voltage reduces due to the increase in load and it is obvious that current will increase according to the load requirement. 


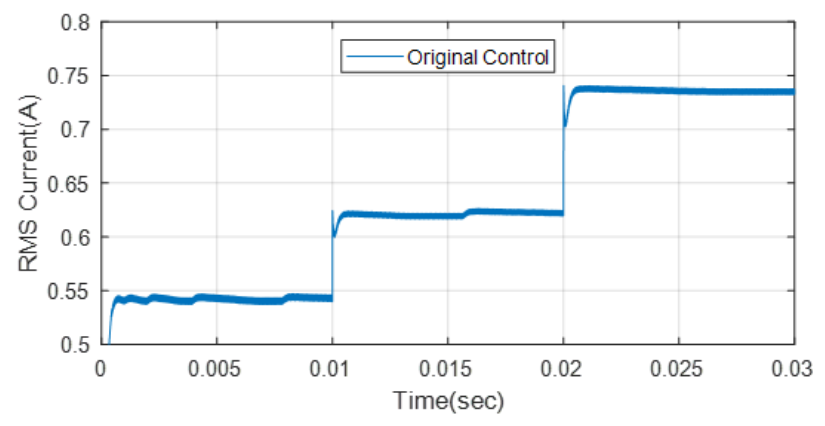

Fig.11: ESU output RMS current for the original controller.

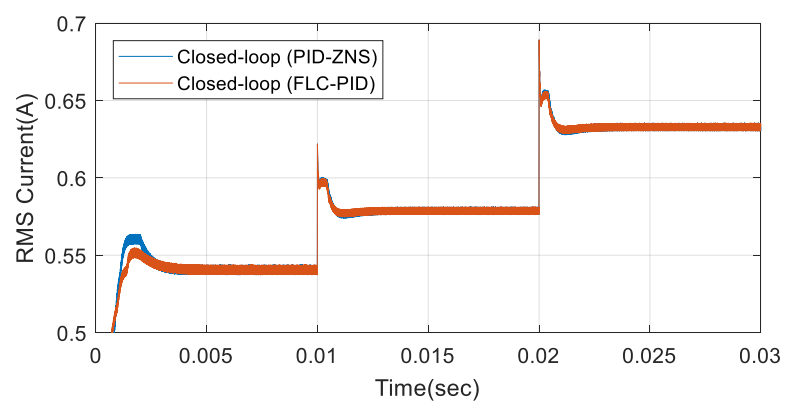

Fig.12: RMS output current for the proposed ESU with PID-ZNS and FLC-PID controller.

In the closed-loop system, the load current increases according to the reduction in voltage. For example, for the original controller, after the second relay is activated, the current increases up to $0.625 \mathrm{~A}$ because there is a slight decrement in the respective voltage. In closed-loop control, the current increases to approximately $0.57 \mathrm{~A}$. This shows how a closed-loop controller is more inclined towards tissue safety than an open-loop controller. The OS\% and STD for the three controllers were shown in Table 2. In Figs. 13, 14 and 15, the power signal for the original controller, for the PID controller tuning methods (ZNS and FLC) and its zoomed version are depicted, respectively. It can be observed from these quantitative figures that improvement in standard deviation using the FLC-PID is $25.8 \%$ more than that of the original controller and $7.4 \%$ more than for the PID-ZNS method. This shows that the increase in power is the highest with the open-loop control method, which implies that this is the most hazardous method among all the three ESU control techniques. A more detailed comparison of the rise time settling time can be seen in Table 2 . It is quite evident from the above analysis and from Table 2 that the closedloop system offers better performance to save the tissue from damage.

\section{Cost of the proposed prototype}

An estimate of the economic advantages of the proposed ESU system can be a valuable indication for hardware implementation. The costs of all components are calculated and mentioned as follows: battery $24 \mathrm{~V}$ is $25 \mathrm{USD}$; DC-DC buck converter is 15 USD; HF inverter is 15 USD; HF transformer is 15 USD; the tissue-equivalent electrical circuit is 25 USD; micro controller is 30 USD; technical fees are 200 USD; other components are 25 USD; shipping charges are 150 USD. Hence, the total cost is 500 USD. The cost of the proposed ESU system is low compared with the low-quality and high-quality commercial ESU. The cost of commercial ESU devices ranges from 4000 USD to 12,000 USD.

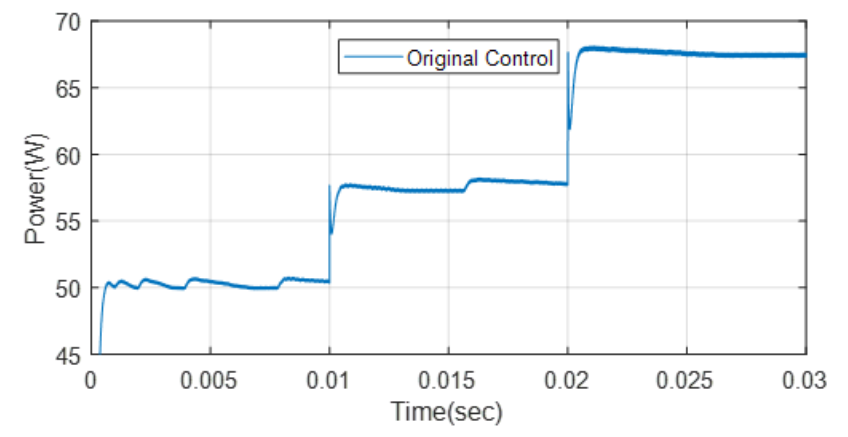

Fig.13: ESU output power for the original controller.

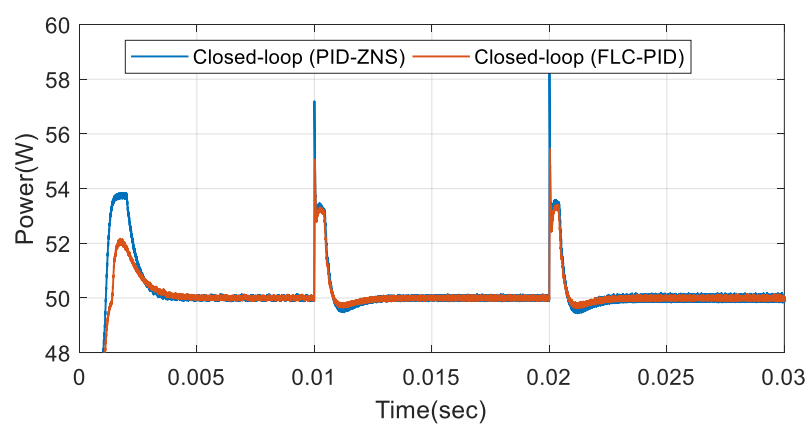

Fig.14: Output power for the proposed ESU with PID-ZNS and FLCPID controller.

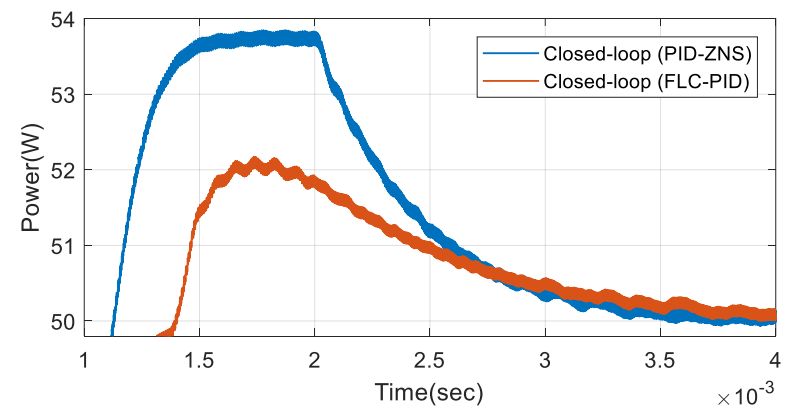

Fig.15: Power signal for the proposed ESU with PID-ZNS and FLCPID controller (Zoomed).

\section{Conclusion}

In this study, a comparative analysis for three types of control techniques are presented to test the performance of electro surgical unit (ESU). The main focus of these controllers was to ensure safety of the patient by reducing the thermal damage to the tissue which is involved in surgical operation. The proposed FLC-PID is proposed with constant power output to keep the stimulation of tissue with the desired electrical power. The controllers were also tested for various Impedance changes to validate the effect of tissue impedance of the human body. From the results it is obvious that, PID with a fuzzy logic tuning method offers better performance as compared to the Ziegler Nichols tuning method. 
Table 2: Summary of results.

\begin{tabular}{|l|c|c|c|}
\hline Performance criteria & $\begin{array}{c}\text { Original } \\
\text { Controller }\end{array}$ & PID-ZNS & PID-FLC \\
\hline Power increasing & $36.20 \%$ & $17.00 \%$ & $10.00 \%$ \\
\hline OS\% of voltage & $0.34 \%$ & $3.70 \%$ & $2.07 \%$ \\
\hline OS\% of current & $0.93 \%$ & $4.26 \%$ & $2.22 \%$ \\
\hline OS\% of Power & $0.80 \%$ & $7.60 \%$ & $4.20 \%$ \\
\hline STD of voltage & $3.38 \%$ & $3.85 \%$ & $3.04 \%$ \\
\hline STD of current & $3.72 \%$ & $4.51 \%$ & $3.39 \%$ \\
\hline STD of Power & $36.40 \%$ & $18.00 \%$ & $10.60 \%$ \\
\hline Tr of voltage & $0.37 \mathrm{~ms}$ & $0.9 \mathrm{~ms}$ & $0.89 \mathrm{~ms}$ \\
\hline Tr of current & $0.37 \mathrm{~ms}$ & $0.9 \mathrm{~ms}$ & $0.89 \mathrm{~ms}$ \\
\hline Tr of power & $0.37 \mathrm{~ms}$ & $0.9 \mathrm{~ms}$ & $0.89 \mathrm{~ms}$ \\
\hline Ts of voltage & unstable & $2.4 \mathrm{~ms}$ & $2.1 \mathrm{~ms}$ \\
\hline Ts of current & unstable & $2.4 \mathrm{~ms}$ & $2.1 \mathrm{~ms}$ \\
\hline Ts of power & unstable & $2.4 \mathrm{~ms}$ & $2.1 \mathrm{~ms}$ \\
\hline \multicolumn{4}{|c|}{$\left(\mathbf{Z}_{\mathbf{1}}\right.$ to $\left.\mathbf{Z}_{\mathbf{2}}\right)$ period $(\mathbf{0}-\mathbf{0 . 0 1})$ sec } \\
\hline Performance criteria & PID-ZNS & PID-FLC \\
\hline Power increasing & $7.60 \%$ & $4.20 \%$ \\
\hline OS\% of voltage & $3.70 \%$ & $2.07 \%$ \\
\hline OS\% of current & $4.26 \%$ & $2.22 \%$ \\
\hline \multicolumn{4}{|l|}{$\left(\mathbf{Z}_{\mathbf{2}}\right.$ to $\left.\mathbf{Z}_{\mathbf{3}}\right)$} \\
\hline
\end{tabular}

Moreover, the cost analysis of the ESU with the proposed controller was also presented and showed that the proposed ESU is economical as compared to commercial devices. Quantitatively, it was found that increase in power for uncertainty in the original controller is $36.2 \%$ while under the proposed controller, the power increment is minimized up to $10.0 \%$. The standard deviation of the power for the original controller is $36.4 \%$, while for the proposed controller, it is $10.6 \%$.

To conclude, among the presented controllers, the proposed controller (PID-FLC) is the best performing controller as it can, more efficiently, maintain a constant value for the output power of the ESU operating inside the patient's body during a surgical operation, which consequently reduces the thermal damage.

\section{Conflict of interest}

Authors state no conflict of interest.

\section{References}

1. H. Sarnago, Ó. Lucía, A. Naval, J. M. Burdío, Q. Castellví, and A. Ivorra, "A versatile multilevel converter platform for cancer treatment using irreversible electroporation," IEEE Journal of Emerging and Selected Topics in Power Electronics, vol. 4, no. 1, pp. 236-242, 2015. https://doi.org/10.1109/JESTPE.2015.2512324
2. A. P. Hu, Y. W. You, F.-Y. B. Chen, D. McCormick, and D. M. Budgett, "Wireless power supply for ICP devices with hybrid supercapacitor and battery storage," IEEE Journal of Emerging and Selected Topics in Power Electronics, vol. 4, no. 1, pp. 273279, 2015. https://doi.org/10.1109/JESTPE.2015.2489226

3. J. A. Sabaté, R. R. Wang, F. Tao, and S. Chi, "Magnetic resonance imaging power: High-performance MVA gradient drivers," IEEE Journal of Emerging and Selected Topics in Power Electronics, vol. 4, no. 1, pp. 280-292, 2015. https://doi.org/10.1109/JESTPE.2015.2474714

4. D. A. Friedrichs, R. W. Erickson, and J. Gilbert, "A new dual current-mode controller improves power regulation in electrosurgical generators," IEEE Transactions on Biomedical Circuits and Systems, vol. 6, no. 1, pp. 39-44, 2011. https://doi.org/10.1109/TBCAS.2011.2159859

5. S. Jensen and D. Maksimovic, "Fast tracking electrosurgical generator using two-rail multiphase buck converter with gan switches," IEEE Transactions on Power Electronics, vol. 32, no. 1, pp. 634-641, 2016. https://doi.org/10.1109/TPEL.2016.2524642

6. A. I. alzaidi, A. yahya, T. T. Swee, and o. Idris, "Development of high frequency generator for bipolar electrosurgical unit," International Journal of Engineering \& Technology, vol. 7, no. 2.29, pp. 20-23, 2018. https://doi.org/10.14419/ijet.v7i2.29.13118

7. F. M. B. Bisinotto, R. A. Dezena, L. B. Martins, M. C. Galvão, J. Martins Sobrinho, and M. S. Calçado, "Burns related to electrosurgery-Report of two cases," Revista Brasileira de Anestesiologia, vol. 67, no. 5, pp. 527-534, 2017. https://doi.org/10.1016/j.bjane.2015.08.018

8. Cordero, Ismael. "Electrosurgical units-how they work and how to use them safely." Community Eye Health, vol. 28, no. 89, pp. 15-16, 2015.

9. K. Bree, S. Barnhill, and W. Rundell, "The dangers of electrosurgical smoke to operating room personnel: a review," Workplace Health \& Safety, vol. 65, no. 11, pp. 517-526, 2017. https://doi.org/10.1177/2165079917691063

10. H. Sarnago, O. Lucia, and J. Burdío, "High performance boost inverter featuring GaN-based devices for electro surgical units," in 2017 IEEE Applied Power Electronics Conference and Exposition (APEC), 2017, pp. 1620-1624: IEEE. https://doi.org/10.1109/APEC.2017.7930916

11. S. D. Schwaitzberg, "Evolutions and revolutions in surgical energy," in The SAGES Manual on the Fundamental Use of Surgical Energy (FUSE): Springer, 2012, pp. 3-13. https://doi.org/10.1007/978-1-4614-2074-3_1

12. K. Dornhof and D. Belik, "An Electrical Circuit for Biological Tissue Simulation in Electrosurgery Models," Biomedical Engineering, vol. 53, no. 1, pp. 40-43, 2019. https://doi.org/10.1007/s10527-019-09874-4

13. V. Bluvshtein, L. Lucke, and M. Widule, "Stabilization of Electrosurgical Cutting Performance Based on Electrode Speed," in 2019 41st Annual International Conference of the IEEE Engineering in Medicine and Biology Society (EMBC), 2019, pp. 166-169: IEEE. https://doi.org/10.1109/EMBC.2019.8856861 
14. D. V. Palanker, A. Vankov, and P. Huie, "Electrosurgery with cellular precision," IEEE Transactions on Biomedical Engineering, vol. 55, no. 2, pp. 838-841, 2008. https://doi.org/10.1109/TBME.2007.914539

15. B. Schneider, E. Dias, and P. J. Abatti, "How can electrosurgical sparks generate undesirable effects?," in 2010 First IEEE Latin American Symposium on Circuits and Systems (LASCAS), 2010, pp. 93-96: IEEE. https://doi.org/10.1109/LASCAS.2010.7410228

16. D. V. Belik and I. N. Kustov, "The System for Measuring the Speed of Movement of the Active Electrode of the Electrosurgical Unit," in 2018 XIV International ScientificTechnical Conference on Actual Problems of Electronics Instrument Engineering (APEIE), 2018, pp. 343-345: IEEE. https://doi.org/10.1109/APEIE.2018.8545753

17. G. Špániková, D. Murgaš, P. Špánik, and M. Frivaldský, "Analysis of critical current field distribution in tissues during electrosurgical procedures," in 2016 ELEKTRO, 2016, pp. 589592: IEEE. https://doi.org/10.1109/ELEKTRO.2016.7512145

18. M. M. Rafiq, M. Ishfaq, M. Ali, A. Ibeas, and J. Herrera, "A closed loop robust control system for electrosurgical generators," in Control Applications for Biomedical Engineering Systems: Elsevier, 2020, pp. 149-168. https://doi.org/10.1016/B978-0-12-817461-6.00006-8

19. E. Yasuno, H. Kato, Y. Kinouchi, and T. Morimoto, "A Fundamental Study on Parameter Estimation of Layerd Local Tissue Impedance for EIT," in 2005 IEEE Engineering in Medicine and Biology 27th Annual Conference, 2006, pp. 66506653: IEEE. https://doi.org/10.1109/IEMBS.2005.1616027

20. P. Imbertson and N. Mohan, "Asymmetrical duty cycle permits zero switching loss in PWM circuits with no conduction loss penalty," IEEE Transactions on Industry Applications, vol. 29, no. 1, pp. 121-125, 1993. https://doi.org/10.1109/28.195897

21. A. J. Mahdi, W. Tang, L. Jiang, and Q. Wu, "A Comparative study on variable-speed operations of a wind generation system using vector control," in International Conference on Renewable Energy and Power Quality (ICREPQ), 2010. https://doi.org/10.24084/repqj08.418
22. K. J. Åström and T. Hägglund, "Revisiting the Ziegler-Nichols step response method for PID control," Journal of Process Control, vol. 14, no. 6, pp. 635-650, 2004. https://doi.org/10.1016/j.jprocont.2004.01.002

23. A. J. Mahdi and M. Aryanezhad, "Fuzzy-Decision-Making Predictive Power Control Approach to On-Grid Photovoltaic Panel", International Journal of Smart Electrical Engineering, vol. 6, no. 01, pp. 21-26, 2017.

24. S. Fahad, N. Ullah, A. J. Mahdi, A. Ibeas, and A. Goudarzi, "An Advanced Two-Stage Grid Connected PV System: A FractionalOrder Controller", International Journal of Renewable Energy Research (IJRER), vol. 9, no. 1, pp. 504-514, 2019.

25. S. Fahad, A. J. Mahdi, W. H. Tang, K. Huang, and Y. Liu, "Particle Swarm Optimization Based DC-link Voltage Control for Two Stage Grid Connected PV Inverter", in 2018 International Conference on Power System Technology (POWERCON), 2018, pp. 2233-2241: IEEE. https://doi.org/10.1109/POWERCON.2018.8602128

26. Dums, José Flavio, Bertoldo Schneider Junior, and Alceu André Badin. "Low cost system to measure active power in electrosurgical units." Research on Biomedical Engineering 33.4 (2017): 313-323. https://doi.org/10.1590/2446-4740.03217

27. Abdullah, A.I., Yahya, A., Rava, M., Swee, T.T., \& Idris, N. (2019). "An Experiment on Thermal Damage Prevention via Power Control in Bipolar Electrosurgical Unit" in International Journal of Engineering and Advanced Technology (IJEAT), ISSN: 2249-8958, Volume-8 Issue-5, June 2019.

28. S. Fahad, N. Ullah, A. J. Mahdi and N. Ullah, " A New Robust Closed Loop Control System for Electrosurgical Generators", Research on Biomedical Engineering (RMBE), 2020. https://doi.org/10.1007/s42600-020-00062-y 